\title{
Hippocampal sleep features: relations to human memory function
}

\author{
Michele Ferrara $^{1}$ *, Fabio Moroni ${ }^{2,3}$, Luigi De Gennaro ${ }^{2}$ and Lino Nobili, ${ }^{4,5}$ \\ 1 Department of Health Sciences, University of L'Aquila, L'Aquila, Italy \\ 2 Department of Psychology, "Sapienza" University of Rome, Roma, Italy \\ ${ }^{3}$ Department of Psychology, University of Bologna, Bologna, Italy \\ ${ }^{4}$ Centre of Epilepsy Surgery "C. Munari," Niguarda Hospital, Milano, Italy \\ ${ }^{5}$ Center of Sleep Medicine, Niguarda Hospital, Milano, Italy
}

\section{Edited by:}

Michael Czisch, Max Planck Institute

of Psychiatry, Germany

\section{Reviewed by:}

Linda J. Larson-Prior, Washington University School of Medicine in St. Louis, USA

Lisa Genzel, Max Planck Institute of

Psychiatry, Germany

\section{*Correspondence:}

Michele Ferrara, Dipartimento di

Scienze della Salute, Università degli

Studi dell'Aquila, Via Vetoio, Coppito 2,

67010 Coppito (L'Aquila), Italy.

e-mail:michele.ferrara@cc.univaq.it
The recent spread of intracranial electroencephalographic (EEG) recording techniques for presurgical evaluation of drug-resistant epileptic patients is providing new information on the activity of different brain structures during both wakefulness and sleep. The interest has been mainly focused on the medial temporal lobe, and in particular the hippocampal formation, whose peculiar local sleep features have been recently described, providing support to the idea that sleep is not a spatially global phenomenon. The study of the hippocampal sleep electrophysiology is particularly interesting because of its central role in the declarative memory formation. Recent data indicate that sleep contributes to memory formation. Therefore, it is relevant to understand whether specific patterns of activity taking place during sleep are related to memory consolidation processes. Fascinating similarities between different states of consciousness (wakefulness, REM sleep, non-REM sleep) in some electrophysiological mechanisms underlying cognitive processes have been reported. For instance, large-scale synchrony in gamma activity is important for waking memory and perception processes, and its changes during sleep may be the neurophysiological substrate of sleep-related deficits of declarative memory. Hippocampal activity seems to specifically support memory consolidation during sleep, through specific coordinated neurophysiological events (slow waves, spindles, ripples) that would facilitate the integration of new information into the pre-existing cortical networks. A few studies indeed provided direct evidence that rhinal ripples as well as slow hippocampal oscillations are correlated with memory consolidation in humans. More detailed electrophysiological investigations assessing the specific relations between different types of memory consolidation and hippocampal EEG features are in order. These studies will add an important piece of knowledge to the elucidation of the ultimate sleep function.

Keywords: hippocampus, stereo-EEG, memory consolidation, local sleep, brain rhythms, oscillations

\section{INTRODUCTION}

The spatial resolution of scalp electroencephalographic (EEG) methods is inadequate to show local physiological processes in the deep brain. However, the recent spread of intracranial EEG recording techniques, mainly used for the presurgical evaluation of drug-resistant epileptic patients, is allowing to partially fill this gap, providing detailed information on the activity of different cortical and subcortical structures during both wakefulness and sleep.

Researchers have mainly focused on the medial temporal lobe (MTL), and in particular on the hippocampal formation, whose peculiar local sleep features have been recently described (for reviews, see Ferrara and De Gennaro, 2011; Nobili et al., 2012). The hippocampal formation plays a central role in the declarative memory formation (Eichenbaum, 2004; Carr et al., 2011). Recent data indicate that sleep contributes to memory formation by consolidating new information and by integrating it with previously stored contents (Diekelmann and Born, 2010). However, the exact neurophysiological mechanisms underlying memory consolidation processes during sleep are still a matter of debate (Girardeau and Zugaro, 2011; Mölle and Born, 2011; Wang et al., 2011).

In this work we will first review available data on the description, obtained by means of invasive deep brain recordings, of the EEG characteristics of hippocampal/parahippocampal sleep. Successively, to establish a connection between subcortical sleep features and memory functions, we will review the available evidence on the relations between hippocampal sleep on the one hand, and sleep mentation (hypnagogic hallucinations and dreams) and memory consolidation after learning on the other.

\section{INTRACORTICAL STUDIES OF HIPPOCAMPAL SLEEP IN HUMANS}

Intracerebral stereo-EEG (SEEG) sleep recordings have recently provided robust support to the idea that sleep is not a spatially global phenomenon, pointing that different cortical and subcortical brain structures may have different and peculiar sleep 
characteristics (Moroni et al., 2007, 2012; Nobili et al., 2011). After the early, sparse and inconclusive data on human sleep features in limbic structures (Brazier, 1968; Wieser, 1984; Mann et al., 1997), a decade ago Bódizs et al. (2001) reported the first "modern" results on hippocampal EEG activity during REM sleep detected by foramen ovale (FO) electrodes in 12 epileptic patients undergoing presurgical examination. FO electrodes allow a mesiotemporal corticography, providing the opportunity to measure hippocampal/parahippocampal activity in a semiinvasive way, without opening the skull. These authors showed for the first time the presence in human REM sleep of a striking, continuous $1.5-$ to $3-\mathrm{Hz}$ rhythmic oscillation in the hippocampal region, while on the scalp the typical REM sleep EEG desynchronization was evident. Slow wave sleep (SWS) was characterized by higher amplitude slow rhythms, with higher power in the lowdelta range $(<1.25 \mathrm{~Hz})$. The $1.5-$ to $3-\mathrm{Hz}$ activity detected with FO electrodes during human REM sleep was considered to be the analog of the hippocampal rhythmic slow activity (RSA) in rodents, although the latter typically falls within the theta range (e.g., O'Keefe and Recce, 1993). This slower tonic activity could be related to a general tendency of larger hippocampal formations to generate slower rhythms. Clearly, although delta activity in hippocampal-parahippocampal derivations seems an intrinsic characteristic of REM sleep, it may have different sources from that of non-REM (NREM) delta oscillation. The latter oscillations result from the thalamocortical system, while it was suggested that the former may be the result of hippocampal-cortical interplay (Bódizs et al., 2001).

Moroni et al. (2007) have successively carried out an extensive and systematic evaluation of hippocampal NREM and REM sleep recorded by intracerebral SEEG directly from the hippocampus. In this study, hippocampal recordings were compared with neocortical and scalp sites aimed to understand whether sleep in the hippocampus shares some basic characteristics with the wellknown scalp recorded sleep. It was shown that delta band exhibits, also in the hippocampus, the typical progressive decrease of power across sleep cycles, indicating that a kind of homeostatic regulation of delta activity is present also in this subcortical structure. Hippocampal sleep also showed some very peculiar, distinctive features during both NREM and REM sleep periods, in particular a lower relative power in the slow oscillation range $(<1 \mathrm{~Hz})$ during NREM sleep compared to the scalp EEG. There was a flattening of the time course of the very low frequencies $(<1 \mathrm{~Hz})$ across sleep cycles as compared to the scalp, with relatively high power even during REM sleep. Finally, a decrease of power in the beta band was found during REM sleep, at variance with the typical increase of power in the cortical recordings.

Together, these findings indicate that during REM sleep the hippocampus shows a generalized tendency to EEG synchronization. In strict agreement with Bódizs et al. (2001), such a synchronization in the low rhythms during REM sleep seems to be the most peculiar feature of hippocampal sleep (Moroni et al., 2007). A prominent mesiotemporal tonic activity during REM sleep in the delta range has been confirmed also by another independent group (Clemens et al., 2009), by means of both visual inspection of the unfiltered recordings and fast Fourier transformation (FFT). This study provided the first evidence during REM sleep of a phase coupling between the tonic oscillations at $1.5-3 \mathrm{~Hz}$ and the gamma activity in human mesiotemporal recordings. An important feature of the rodent hippocampal RSA is that it co-occurs with high-frequency gamma activity such that the amplitude of the gamma activity is modulated by the phase of the theta cycle (Bragin et al., 1995; Chrobak and Buzsáki, 1998). Therefore, the above results (Clemens et al., 2009) support the hypothesis that the oscillatory delta activity in human REM sleep may be the analog of the animal hippocampal theta.

Finally, Cantero et al. (2003) reported the first, and to date the only, evidence of the presence of human theta oscillations (4$7 \mathrm{~Hz}$ ) within the hippocampus. These oscillations, recorded by bipolar multicontact depth electrodes, appeared in short bursts during REM sleep, while no similar electrophysiological events were observed in either SWS or wakefulness. The hippocampus and neocortical regions were uncoupled during the emergence of theta oscillations, suggesting that these cerebral structures have distinct and independent theta-wave generators. Notably, there was no evidence for phase coupling of gamma activity with the observed theta oscillations (Cantero et al., 2003). The absence of gamma-theta coupling provide a further indication that delta rather than theta activity - should be considered as the human analog of the animal hippocampal RSA during REM sleep.

It is of note that recent observations from our laboratory lend further support to this view, confirming and extending the above findings (Bódizs et al., 2001; Moroni et al., 2007; Clemens et al., 2009). In particular, we have confirmed the presence of delta activity during REM sleep in the human, and provided the first evidence of its existence in the spontaneous SEEG also during wakefulness (Moroni et al., 2012, see below for more detailed discussion).

\section{HIPPOCAMPAL-NEOCORTICAL DIALOG AND MEMORY CONSOLIDATION}

Oscillatory dynamics within and between cerebral regions are implicated in waking cognitive processes such as memory and perception, for instance in the gamma activity range (e.g., Fell et al., 2001). Therefore, it is relevant to assess their relations with the drastic changes in cognitive features, typically observed in different brain states. Indeed, specific oscillations and phasic sleep events, such as slow waves, spindles, and ripples, are candidate to be a neurophysiological mechanism for information transfer between hippocampus and neocortex underlying sleep-related memory consolidation (Axmacher et al., 2006, 2009; Fell and Axmacher, 2011).

Slow oscillations $(0.5-1.0 \mathrm{~Hz})$ arise in the neocortex but spread to many other areas including the hippocampus and thalamus (Massimini et al., 2004; Nir et al., 2011), and fulfill an important temporal gating function. The slow oscillation comprises a hyperpolarization phase or down-state, during which virtually all cortical neurons are deeply hyperpolarized, and a depolarization phase or up-state. During the up-state, when neurons fire at rates that are even higher than in quiet wakefulness (Steriade et al., 2001), sleep spindles (faster and lower amplitude oscillations in the 11- to 15$\mathrm{Hz}$ range) occur in the thalamus and travel to neocortical areas (for a review, De Gennaro and Ferrara, 2003). Thalamocortical spindles stimulate early gene expression and glutamate receptors thereby creating optimal conditions for long-term potentiation (LTP) in 
the neocortex (for a review, Born et al., 2006). At the same time, high-frequency ripples at $100-300 \mathrm{~Hz}$ and sharp waves occur in the hippocampus. These events are associated with the offline replay of neural sequences learned during encoding (e.g., Lee and Wilson, 2002; Ji and Wilson, 2007). Although many evidences confirm a role of slow oscillations, spindles, and sharp wave-ripple complexes in memory consolidation during sleep, how they interact to subserve a transfer of information is still debated.

\section{STATE DEPENDENT CHANGES IN LOCAL AND LONG-RANGE (HIPPOCAMPO-NEOCORTICAL) RELATIONS}

Oscillations in the gamma frequency band typically increase in brain states characterized by active cognitive processing, whether of external percepts or internal thoughts and images (Llinas and Ribary, 1993). Cantero et al. (2004) assessed the hypothesis that high levels of gamma coherence between neocortex and hippocampus facilitate the high-level cognitive processes typical of wakefulness, whereas low gamma coherence characterizes those states where higher cognitive processes are strongly diminished, as during sleep (Cantero et al., 2004). They indeed reported higher levels of gamma coupling within and between cortical areas during wakefulness than during SWS and REM sleep, when gamma coherence was negligible. Additionally, gamma functional coupling between the neocortex and hippocampus was dramatically decreased during both SWS and REM sleep compared to wakefulness. According to the authors, the lack of cross-talk within and between different brain regions in the gamma range during both REM and SWS, well correlates with brain states characterized by a reduction in attention, memory, and perceptive functions, and an inability to create a global awareness of the world or the self (Cantero et al., 2004). These results lend support to the hypothesis that changes in large-scale correlative synchrony in gamma activity are the neurophysiological substrate of reduced cognitive functions during sleep. Along the same line, Fell et al. (2003) analyzed the intracranial EEG activity recorded from the rhinal cortex and the hippocampus to assess whether measures quantifying rhinal-hippocampal interaction may explain the impaired declarative memory during sleep. They reported an overall reduction of rhinal-hippocampal coherence during sleep compared to wakefulness, particularly pronounced in the upper gamma band $(28-44 \mathrm{~Hz})$. These data indirectly support the hypothesis that a coherence decrease may be the electrophysiological substrate of the sleep-related reduction of declarative memory (Fell et al., 2003).

In recent years, some authors have focalized their attention on specific hippocampal sleep features, like sharp wave-ripples, also in coincidence with other phasic events recorded in the neocortex or over the scalp. Ripples are an important hippocampal highfrequency activity originated in the CA1 region of the hippocampus and occurring in association with sharp waves emerging from CA3 (Buzsáki, 1986). Fast ripple oscillations play an active role in plasticity processes. Indeed, sharp waves induce LTP in hippocampal CA1 pyramidal neurons and sharp wave-ripple complexes in brain slices in vitro can be generated by plasticity-inducing stimulation paradigms (Behrens et al., 2005; for a review, Axmacher et al., 2009). Moreover, it has been shown that selective elimination of sharp wave-ripples during post-training consolidation periods (Girardeau et al., 2009) or disruption of neuronal activity during ripple events impairs spatial learning (Ego-Stengel and Wilson, 2010). These results establish a causal link between sharp waveripples and memory consolidation. However, the question of how exactly replay would participate in memory formation remains open (Wang et al., 2011).

Sleep spindles and ripples are concentrated on the depolarizing phase of neocortical slow activity (Mölle et al., 2002), suggesting that these coordinated patterns of activity take place during a highly responsive and plastic state of the neocortex (Steriade, 2003; Steriade and Timofeev, 2003). The temporal synchronization of thalamocortical spindles and hippocampal sharp waves by slow oscillations is believed to ensure that sequence replay in the hippocampus coincides with neocortical readiness for encoding (Born et al., 2006). This provides a potential physiological mechanism for offline consolidation of hippocampal-based memories during sleep (for reviews, Born, 2010; Mölle and Born, 2011).

Human ripples share many characteristics with those in rodents (Siapas and Wilson, 1998), although they show a lower frequency $(80-140 \mathrm{~Hz})$. Clemens et al. (2007) using parahippocampal FO electrodes recordings, evaluated the hypothesis that a similar coupling between these oscillatory phenomena does exist also in humans, providing the first evidence for a temporal coupling between parahippocampal ripples, sleep spindles, and slow oscillations. In particular, the neocortical slow oscillation induced a grouping effect such that spindle and ripple activity were decreased during the negative slow oscillation half-wave (down-state), and increased during the subsequent positive up-state. Interestingly, the synchronization of ripples to the slow oscillation was clear only in the patients whose MRI scans did not indicate severe structural alterations in mesiotemporal regions. A temporal association between ripple and spindle activity was also reported: ripple activity was increased shortly before spindle peaks and decreased distinctly after the peak (Clemens et al., 2007). These findings are thus consistent with the notion of a hippocampalto-neocortical information transfer during sleep that is linked to coordinated ripple and spindle activity synchronized to cortical slow oscillations (Clemens et al., 2007). Recently, Nir et al. (2011) demonstrated that neurons in entorhinal cortex, hippocampus, and amygdala modulate their spiking activities in concert with EEG slow waves. In particular they revealed a robust tendency of sleep slow waves to propagate from medial prefrontal cortex to the MTL and hippocampus. They also confirmed that hippocampal ripples tend to occur around the up-state of slow waves (see Molle et al., 2006; Clemens et al., 2007). Moreover, they showed delayed and attenuated spike discharges in entorhinal cortex compared with hippocampus, a finding that support the notion that ripples reflect local hippocampal output.

However, while these findings show that hippocampal ripples, neocortical sleep spindles, and up-states are locked in time, unfortunately they do not unequivocally reveal the direction of the hippocampo-cortical or cortico-hippocampal information transfer (Axmacher et al., 2009).

Indirect evidence about the relevance of a co-occurrence of slow and fast hippocampal oscillations for the processing of declarative memories comes from a correlational study comparing surface versus mediotemporal recordings (Poepel et al., 2007). It was shown that the typical negative correlation of slow 
and fast frequency bands in scalp EEG reverses into a positive correlation in hippocampal recordings, particularly during NREM sleep. These results are in line with earlier data suggesting that the co-occurrence of fast and slow oscillations is important for memory processing in the hippocampus during wakefulness (Lisman and Idiart, 1995; Jensen and Lisman, 2005).

More recently Clemens et al. (2011) assessed, with parahippocampal FO recordings, the temporal relationship between parahippocampal ripple activity $(80-140 \mathrm{~Hz})$ and spindles recorded from frontal, parietal, and parahippocampal regions. Based on findings in animals, it was hypothesized that ripples originating from the hippocampus combine with spindles to provide a fine-tuned temporal frame for a persistent transfer of memoryrelated information to the neocortex (Siapas and Wilson, 1998; Marshall and Born, 2007). Spindles were indeed coupled with parahippocampal ripples such that, during parietal and parahippocampal spindles, the ripple activity was higher before than after the spindle peak. On a finer timescale, parahippocampal ripple activity was phase-locked to parahippocampal and parietal spindle troughs (Clemens et al., 2011). These data are consistent with the idea of a temporally fine-tuned hippocampus-to-neocortex transfer of information taking place during NREM sleep.

Further support to the hypothesis of an information flow from the hippocampus to the neocortex comes from another recent large-scale microelectrode recording study (Le Van Quyen et al., 2010). The authors reported evidence of a probabilistic coupling between parahippocampal gamma oscillations and hippocampal ripple/sharp wave complexes. In particular, cortical gamma oscillations tended to occur within $100 \mathrm{~ms}$ after hippocampal ripple/sharp waves, suggesting an information flow from the hippocampus to the cortex. It was speculated that these highfrequency patterns briefly restore "microwake" activity and are important for consolidation of memory traces acquired during previous awake periods.

On the other hand, a completely different pattern of interactions between neocortex and hippocampus during human sleep emerges from a study investigating directional coupling in intracranial EEG data using a phase-modeling approach (Wagner et al., 2010). Unexpectedly, the influence of neocortical regions on the hippocampus significantly increased during sleep as compared to waking state. Similarly, Nir et al. (2011) provided evidence showing that during NREM sleep neural activity propagates predominantly from the neocortex to the hippocampus. They also reported that ripples represent spatially constrained hippocampal output, with entorhinal cortex showing a delayed transient peak following the hippocampus, whereas a transient firing in medial prefrontal cortex units was not found following ripple occurrence.

These results question the standard model of hippocampalneocortical interactions, which postulates the existence of simple unidirectional influences inverting between waking and sleep states (e.g., Buzsáki, 1989, 1998). Instead, they suggest that sleepdependent consolidation may be accomplished by an active retrieval of hippocampal information by the neocortex, since the neocortex seems to exert an increasing influence onto structures of the MTL toward deeper sleep stages. The observed directional changes may be interpreted in keeping with the proposal of co-activation of neocortex and hippocampus during NREM sleep (Ji and Wilson, 2007; Mehta, 2007), suggesting that a coordinated replay in both structures could be an important feature of memory consolidation. According to this model, newly learned memory traces are integrated into neocortical regions during coactivation, while at the same time recently learned information are erased in the hippocampus (Mehta, 2007). Future studies could critically clarify this issue by directly relating directional coupling to memory performance assessed before and after sleep.

Finally, we have recently observed that two different types of delta activity are originated in the hippocampus (Moroni et al., 2012). The first is a low-delta rhythm $(0.5-2.0 \mathrm{~Hz})$, which appears as a transient (phasic) rhythm present during all the vigilance states, but intriguingly synchronous between the hippocampi mainly during the activated states (wake and REM sleep). The second is a high-delta rhythm $(2.0-4.0 \mathrm{~Hz})$, which instead appears as an oscillatory background hippocampal rhythm present during all the states, but only weakly in phase between hemispheres, as showed by coherence analysis. In other words, both left and right hippocampi produce slow EEG rhythms during NREM sleep, but they appear to be out of phase. These data can be interpreted in the framework of the sequential hypothesis of sleep consolidation (Giuditta et al., 1995). According to the hypothesis, we can differentiate between a system consolidation, mainly dependent on SWS and involving widespread brain areas, and a local synaptic plasticity referred to REM sleep. SWS system consolidation engages wide cortical and hippocampal circuits that could explain the loss of coherence between hippocampi observed in our data. In other words, the neocortical slow oscillation would facilitate a long-range transfer of (mainly declarative) information (see Diekelmann and Born, 2010). On the other hand, the disentangled, localized nature of synaptic consolidation during REM sleep could explain the rise of inter-hippocampal network synchronization in the low-delta range observed by Moroni et al. (2012). These novel observations prompt further studies focusing on task-dependent SEEG activity to shed light on the possible functional differences between slow and fast hippocampal delta rhythms in relation to learning.

\section{HIPPOCAMPAL SLEEP FEATURES AND SLEEP MENTATION}

A possible approach to the study of memory formation during sleep is the analysis of dream recall. During wakefulness, the formation of declarative memories is accompanied by the phase synchronization of rhinal-hippocampal EEG activity in the gamma range and an increased EEG coherence in the lowfrequency range (e.g., Fell et al., 2001). It has been suggested that dreams are hardly remembered upon awakening because declarative memory is disfacilitated during sleep (Hobson and Pace-Schott, 2002). Consistent with this hypothesis, during sleep a reduced rhinal-hippocampal and intrahippocampal EEG coherence has been observed compared to wakefulness, particularly within the gamma range (Fell et al., 2003). Therefore, Fell et al. (2006) hypothesized that the interindividual differences in the ability to build declarative memories during sleep could be related to variations in mediotemporal connectivity. To this aim, 12 epileptic patients, stereotactically implanted with multicontact depth electrodes along the longitudinal axis of each mediotemporal lobe, were awakened after at least $5 \mathrm{~min}$ from the onset 
of the second and of the following REM periods, and asked whether they remembered having had a dream and whether they could recall any specific content. Coherence analyses showed that rhinal-hippocampal connectivity values were approximately twice as large for patients with good dream recall compared to those patients with poor recall. This suggests that rhinal-hippocampal connectivity may be a crucial factor in determining declarative memory formation. In particular, a successful memorization of dreams is accompanied by an enhanced rhinal-hippocampal and intrahippocampal EEG coherence, not an EEG power increase within mediotemporal structures, pointing out a common neurophysiological mechanism between wakefulness and sleep (Fell et al., 2001, 2006). Shared electrophysiological mechanisms underlying the encoding and recall of episodic memories between different states of consciousness have been suggested also by a recent study of scalp EEG topography (Marzano et al., 2011). Interestingly, dreaming about a learning task during NREM sleep is associated with a further sleep-related improvement on a hippocampal spatial memory task (Wamsley et al., 2010). This recent finding indirectly supports the notion that a high rhinal-hippocampal and intrahippocampal connectivity may affect both dreaming experience and declarative memory consolidation, leading to higher dream recall rates and an improved performance.

REM sleep shares many physiological and psychological features with the wake-sleep transition, such as the similarities of the scalp recorded EEG of the two states. Moreover, the vivid hypnagogic imagery during sleep onset owns some of the features of REM sleep dreaming. It is interesting to note that REMlike $1.5-3.0 \mathrm{~Hz}$ hippocampal/parahippocampal activity increased during the transition from wakefulness to sleep, reaching an average of $82 \%$ of tonic REM just shortly after the first electrophysiological signs of sleep (Bódizs et al., 2005). The REM-like 1.5-3.0 Hz hippocampal/parahippocampal activity observed during the wake-sleep transition in this study supports the covertREM sleep hypothesis of dreaming, which suggests that "hidden" REM sleep features are present during sleep onset, explaining the vivid hypnagogic imagery (Nielsen, 2000). Intriguingly, the two sleep states accompanied by the most vivid imagery (sleep onset and REM sleep) are indeed characterized by a similar hippocampal/parahippocampal slow activity pattern.

Altogether, these studies indirectly support the existence of a relationship between memory consolidation and hippocampalparahippocampal electrical activity during sleep in humans.

\section{HIPPOCAMPAL SLEEP FEATURES AND LEARNING}

According to the active system consolidation model, during NREM sleep the newly acquired memory traces are repeatedly reactivated, which stimulates the gradual redistribution of these memories such that synaptic connections within the neocortex are strengthened, forming more persistent memory representations (Mölle and Born, 2011). Such a dialog between hippocampus and neocortex during sleep is brought about by different coordinated local phasic events and oscillations (cortical slow oscillations, hippocampal ripples, thalamic spindles). Are there any direct evidence relating the acquisition of new information to modifications of hippocampal sleep patterns or indexes of hippocampal-neocortical dialog?
To date, only three studies have specifically investigated the effects of the pre-sleep acquisition of new information (i.e., learning) on hippocampal-parahippocampal electrical activity during sleep in humans, providing some direct electrophysiological evidence of memory consolidation occurring during sleep.

In the first study, Axmacher et al. (2008a) recorded hippocampal event-related potentials (ERPs), by means of intracranial EEG from the hippocampus and rhinal cortex of nine epileptic patients while they retrieved information acquired either before or after a nap in the afternoon or on a control day without nap. Results indicated that a nap facilitates memory consolidation during postsleep waking state. Moreover, the authors reported that the amount of delta activity during the nap was positively correlated with individuals' performance in a declarative task. However, on the basis of the hippocampal ERP responses, the authors suggested that memory consolidation may occur also during waking state.

In the second study, the same group (Axmacher et al., 2008b) reported the first direct behavioral evidence that ripples are correlated with memory consolidation in humans. Intracranial electroencephalogram was recorded with macroelectrodes from the hippocampus and rhinal cortex in 11 epilepsy patients during a memory consolidation task carried out before and after an afternoon $1 \mathrm{~h}$ nap. Results from cross-correlation analysis revealed that ripples in the hippocampus occurred significantly earlier than in the rhinal cortex. Furthermore, hippocampal ripples were significantly locked to the phase of hippocampal delta band activity, occurring close to the positive peak of delta waves. Interestingly, ripples occurred with the highest incidence during periods when subjects laid awake during the nap time. The number of rhinal, but not hippocampal, ripples was correlated with the number of successfully recalled items after the nap. In humans, a similar relationship was already reported between parahippocampal ripples and neocortical slow oscillations (Clemens et al., 2007). Based on these findings, the authors proposed that the coupling between neocortical slow oscillations and hippocampal ripples may result from a phase-locking of ripples to hippocampal delta band activity, which is itself phase-locked to neocortical slow oscillations (Axmacher et al., 2008b).

The third study (Moroni et al., 2008) reported the first direct evidence that slow hippocampal oscillations are involved in human memory consolidation. In this study, we investigated the effects of both procedural and declarative learning on the qualitative and quantitative measures of sleep recorded from the hippocampus in eight epileptic patients undergoing presurgical evaluations. We reasoned that, if the hippocampus is directly involved in the memory consolidation process, we should expect significant changes in the hippocampal SEEG slow rhythms, as suggested by the functional link between sleep EEG low frequencies and post-learning plasticity (e.g., Aeschbach et al., 2008). We showed that a short but intensive training on a sequential finger tapping task, but not on a paired associate word learning task, is followed by an increase in the amount of scalp recorded SWS. This effect was paralleled by an increase in the hippocampal SEEG power in the very low-frequency range $(0.5-1.0 \mathrm{~Hz})$ during the first post-learning NREM period. The magnitude of procedural performance improvement was significantly correlated with the subsequent SEEG low-frequency power increase, which supports 
the existence of a direct link between procedural memory consolidation and very low hippocampal SEEG rhythms. Interestingly, at variance with previous scalp EEG studies (e.g., Huber et al., 2004), the effect was strictly limited to the very low-frequency range $(0.5-1.0 \mathrm{~Hz})$. These different outcomes may be related to the functional dissociation observed in the delta band during hippocampal sleep (Moroni et al., 2007, 2012). If this is the case, the low-delta band may reveal an independent behavior in the hippocampus compared to the neocortex. Therefore, within the hippocampus, the effects of plasticity on sleep may be limited to the very low frequencies rather than the entire delta range. Consistent with these observations, in vitro stimulation paradigms of hippocampal cells indicated that stimulations at $<1 \mathrm{~Hz}$ are ideally suited to induce depotentiation or depression of synaptic transmission (Kemp and Bashir, 2001). Remarkably, in humans the overnight improvement in another procedural task performance (texture discrimination) was maximally correlated with the lowest EEG frequencies $(0.75-1.00 \mathrm{~Hz})$ within the delta range (Aeschbach et al., 2008). Similarly, Massimini et al. (2009) reported that the activity within the $0.75-$ to $1.5-\mathrm{Hz}$ frequency range reacts most sensitively to experimental manipulations affecting synaptic plasticity. Our findings (Moroni et al., 2008) can be also interpreted according to the Synaptic Homeostasis hypothesis (Tononi and Cirelli, 2006), which posits that slow oscillations during sleep, with the sequence of depolarization (up-phase) and hyperpolarization (down-phase) at a cellular level, are associated with the local processes of post-learning synaptic downscaling. In this view, sleep serves predominantly to reduce synaptic weights and to reset the system for the optimal encoding of new information after sleep. Hippocampal slow oscillations during sleep may be therefore associated with the local processes of post-learning synaptic downscaling.

Finally, Bódizs et al. (2002) explored the relation between individual-specific (trait-like) hippocampal sleep signs and cognitive functions, observing that slow activity (below $1.25 \mathrm{~Hz}$ ) at the parahippocampal region during both SWS and phasic REM sleep were positively correlated with visual memory and verbal learning task performance. Therefore, these findings suggest that sleep-dependent, phase-specific hippocampal synchronized slow activity $(<1.25 \mathrm{~Hz})$ may be an index of waking memory functioning.

\section{CONCLUSION AND FUTURE AVENUES}

In recent years, the study of human cortical and subcortical sleep by means of invasive EEG recordings have provided fascinating data that have moved forward our knowledge of sleep mechanisms and offered hints about sleep functions in relation to memory. An important caveat in studies using intracranial EEG recordings is the peculiar study population. Only patients with a long history of pharmacoresistant epilepsy are evaluated for surgical purposes. It remains unclear whether or not many years of epilepsy may damage the brain or cause it to use alternative mechanism for memory consolidation. Although data are typically recorded during seizure-free periods, inter-ictal epileptiform activity, as well as antiepileptic drugs (AED) could affect sleep in general, and the nature of slow waves in particular. Nevertheless, it is of note these results have been consistently reported in different studies, despite drastically different clinical profiles and AED treatments in patients characterized by distinct site of origin of the seizures.

One of the most intriguing findings is the similarity between different states of consciousness (wakefulness, REM sleep, NREM sleep) in some electrophysiological mechanisms underlying cognitive processes such as the encoding and recall of episodic memories. For instance, oscillatory dynamics in the gamma EEG activity range within and between cerebral regions are implicated in waking memory and perception processes (e.g., Fell et al., 2001), but they also seem important to explain some of the cognitive features of sleep. In fact, coherence in the gamma range is hampered during sleep (Fell et al., 2003; Cantero et al., 2004); consistently, a successful recall of dreams is possible only in presence of an enhanced rhinal-hippocampal and intrahippocampal EEG coherence (Fell et al., 2006). Therefore, changes in local and/or in large-scale synchrony in gamma activity may be the neurophysiological substrate of the deficit of declarative memory during sleep.

As far as the relations between sleep and learning are concerned, the above reviewed findings suggest two different (not mutually exclusive) roles for sleep in memory consolidation.

First, sleep seems to specifically support information transfer through specific coordinated neurophysiological events (slow waves, spindles, ripples) that would facilitate the integration of new information into the pre-existing cortical networks (Diekelmann and Born, 2010; Mölle and Born, 2011). Adopting the widely used two-stage model of memory consolidation, in which encoding first takes place within a fast hippocampal store and is subsequently transferred offline to the neocortex over time (McClelland et al., 1995; Frankland and Bontempi, 2005), it is argued that slow oscillations play an essential role in coordinating this transfer. A second idea, the multiple trace theory (Nadel and Moscovitch, 1997), supposes that multiple traces of single-trial episodic events are encoded in both cortex and hippocampus. Recent observations in rodents (Tse et al., 2011) fits with this idea, and challenge the concept of distinct fast (hippocampal) and slow (cortical) learning systems, shedding light on the neural mechanisms of memory assimilation into schemas.

A schema is a cognitive structure that represents organized knowledge of the world formed through the implicit abstraction of regularities within the environment. According to another model (Lewis and Durrant, 2011), the overlapping reactivation of related memories during SWS selectively strengthens shared elements. Repeated reactivations progressively builds schematic representations of the relationships between stimuli, leading to the addition of new knowledge to existing schemata.

Within the first theoretical framework, the ripples-spindles coupling (Clemens et al., 2011) and the synchronization of ripples and spindles to the slow oscillation (Clemens et al., 2007) are consistent with the hypothesis that the interaction between hippocampal and thalamocortical networks provides a temporally fine-tuned framework for hippocampus-to-neocortex transfer of information during SWS, underlying the consolidation of hippocampus-dependent memories during sleep (Buzsáki, 1989, 1998; Gais and Born, 2004; Born et al., 2006).

However, recent evidence has been provided that does not fit the theory. For example, the above mentioned study on directionality 
of coupling in intracranial EEG have questioned this model (Wagner et al., 2010), reporting that the influence of neocortical regions on the hippocampus significantly increased during sleep as compared to waking state. Consistently, Nir et al. (2011) showed that most sleep slow waves and the underlying active and inactive neuronal states occur locally. In any case, slow waves usually propagate from medial prefrontal cortex to the MTL and hippocampus. Therefore, future studies should clarify this issue by directly assessing directional coupling during sleep before and after successful learning.

A second role for sleep is to restore saturated hippocampal plasticity (Moser et al., 1998; Tononi and Cirelli, 2006) and allow a better and more rapid processing through the hippocampus after sleep. Recent neuroimaging data provided support to this idea, indicating that deep sleep preceding a task optimizes the hippocampus for encoding of novel information (Van Der Werf et al., 2009).

To date, only few studies provided direct evidence that rhinal ripples as well as slow hippocampal oscillations are correlated with memory consolidation in humans (Axmacher et al., 2008b; Moroni et al., 2008). As far as the mechanism underlying this process is concerned, it may be that the coupling between neocortical slow oscillations and hippocampal ripples may result from a phase-locking of ripples to hippocampal delta band activity, which is itself phase-locked to neocortical slow oscillations (Axmacher et al., 2008b). However, more detailed electrophysiological investigations assessing the specific relationships between different types of memory consolidation and hippocampal EEG features are in order, also taking into account the specific roles of different sleep stages.

Finally, it should be clarified whether sleep-dependent memory consolidation is accompanied by a hippocampo-to-neocortical information transfer, as suggested by some authors (e.g., Buzsáki, 1989, 1998), or by a coordinated replay in both neocortex and hippocampus (Ji and Wilson, 2007; Mehta, 2007; Wagner et al., 2010). In keeping with the sequential hypothesis of memory consolidation during sleep (Giuditta et al., 1995), it is also possible that a system consolidation, mainly dependent on SWS and involving widespread brain areas, may coexists with a local synaptic plasticity restricted to REM sleep (Diekelmann and Born, 2010). In that case, these distinct processes would be clearly dependent

\section{REFERENCES}

Aeschbach, D., Cutler, A. J., and Ronda, J. M. (2008). A role for non-rapideye-movement sleep homeostasis in perceptual learning. J. Neurosci. 28, 2766-2772.

Axmacher, N., Draguhn, A., Elger, C. E., and Fell, J. (2009). Memory processes during sleep: beyond the standard consolidation theory. Cell. Mol. Life Sci. 66, 2285-2297.

Axmacher, N., Haupt, S., Fernandez, G., Elger, C. E., and Fell, J. (2008a). The role of sleep in declarative memory consolidation - direct evidence by intracranial EEG. Cereb. Cortex 18, 500-507.

Axmacher, N., Elger, C. E., and Fell, J. (2008b). Ripples in the

on different electrophysiological mechanisms that are to be further clarified.

To further complicate the picture, there is evidence suggesting that SWS is characterized by local phenomena, more than by the facilitation of long-distance connections. Spoormaker et al. (2010), for example, in an fMRI study of functional connectivity showed that such connections are sharply reduced in SWS. This finding was substantially replicated by a functional connectivity analysis of EEG data that demonstrated diffuse localized cortical interactions throughout much of the cortex during the waking state; localized interactions appeared to lose strength in favor of more distant interactions during REM sleep, while SWS only revealed a localized cluster of left fronto-temporal connectivity (Langheim et al., 2011).

Based on these findings, it may be hypothesized that the largescale functional brain network in SWS moved toward a regular network (Watts and Strogatz, 1998). This network organization indeed provides a functional brain state optimal for reprocessing information in segregated functional systems, which is more in accord with a memory-reprocessing hypothesis than with a memory-transfer hypothesis of SWS (Spoormaker et al., 2011). Moreover, this kind of local functioning contrasts with the active system consolidation model, which assumes that SWS enable an effective hippocampus-to-neocortex transfer of the reactivated information (Diekelmann and Born, 2010; Mölle and Born, 2011). Accordingly, Nir et al. (2011) reported that hippocampal sharp wave-ripples hardly reach the entorhinal cortex, which would speak against the theory of their role in memory transfer.

Future studies focusing on task-dependent changes of hippocampal-neocortical activity and connectivity will possibly shed light on these intriguing issues, and add an important piece of knowledge to the elucidation of the ultimate sleep function.

\section{ACKNOWLEDGMENTS}

Michele Ferrara has been supported by grants from the Compagnia di San Paolo, Programma Neuroscienze 2008/09 (3896 SD/sd, 2008.2130) and the University of L'Aquila (Ricerche di Ateneo ex $60 \%$ ). Fabio Moroni has been supported by a grant from ESRS Sanofi-Aventis Research Grant 2008/10. Luigi De Gennaro has been supported by a grant from the Compagnia di San Paolo, Programma Neuroscienze 2008/09 (3889 SD/sd, 2008.1300).

humans. Neurobiol. Learn. Mem. 78, 441-457.

medial temporal lobe are relevant for human memory consolidation. Brain 131, 1806-1817.

Axmacher, N., Mormann, F., Fernandez, G., Elger, C. E., and Fell, J. (2006). Memory formation by neuronal synchronization. Brain Res. Rev. 52, 170-182.

Behrens, C. J., van den Boom, L. P., de, H. L., Friedman, A., and Heinemann, U. (2005). Induction of sharp waveripple complexes in vitro and reorganization of hippocampal networks. Nat. Neurosci. 8, 1560-1567.

Bódizs, R., Bekesy, M., Szucs, A., Barsi, P., and Halasz, P. (2002). Sleep-dependent hippocampal slow activity correlates with waking memory performance in
Bódizs, R., Kantor, S., Szabo, G., Szucs, A., Eross, L., and Halász, P. (2001). Rhythmic hippocampal slow oscillation characterizes REM sleep in humans. Hippocampus 11, 747-753.

Bódizs, R., Sverteczki, M., Lázár, A. S., and Halász, P. (2005). Human parahippocampal activity: non-REM and REM elements in wake-sleep transition. Brain Res Bull. 65, 169-176.

Born, J. (2010). Slow-wave sleep and the consolidation of long-term memory. World J. Biol. Psychiatry 11, 16-21.

Born, J., Rasch, B., and Gais, S. (2006). Sleep to remember. Neuroscientist 12, 410-424.
Bragin, A., Jandó, G., Nádasdy, Z., Hetke, J., Wise, K., and Buzsáki, G. (1995). Gamma (40-100 Hz) oscillation in the hippocampus of the behaving rat. J. Neurosci. 15, 47-60.

Brazier, M. A. (1968). Studies of the EEG activity of limbic structures in man. Electroencephalogr. Clin. Neurophysiol. 25, 309-318.

Buzsáki, G. (1986). Hippocampal sharp waves: their origin and significance. Brain Res. 398, 242-252.

Buzsáki, G. (1989). Two-stage model of memory trace formation: a role for "noisy" brain states. Neuroscience 31, 551-570.

Buzsáki, G. (1998). Memory consolidation during sleep: a 
neurophysiological perspective. $J$. Sleep Res. 7, 17-23.

Cantero, J. L., Atienza, M., Madsen, J. R., and Stickgold, R. (2004). Gamma EEG dynamics in neocortex and hippocampus during human wakefulness and sleep. Neuroimage 22, 1271-1280.

Cantero, J. L., Atienza, M., Stickgold, R., Kahana, M. J., Madsen, J. R., and Kocsis, B. (2003). Sleep-dependent theta oscillations in the human hippocampus and neocortex. J. Neurosci. 23, 10897-10903.

Carr, M. F., Jadhav, S. P., and Frank, L. M. (2011). Hippocampal replay in the awake state: a potential substrate for memory consolidation and retrieval. Nat. Neurosci. 14, 147-153.

Chrobak, J. J., and Buzsáki, G. (1998). Gamma oscillations in the entorhinal cortex of the freely behaving rat. J. Neurosci. 18, 388-398.

Clemens, Z., Molle, M., Eross, L., Barsi, P., Halasz, P., and Born, J. (2007). Temporal coupling of parahippocampal ripples, sleep spindles and slow oscillations in humans. Brain 130, 2868-2878.

Clemens, Z., Mölle, M., Eross, L., Jakus, R., Rásonyi, G., Halász, P., and Born, J. (2011). Fine-tuned coupling between human parahippocampal ripples and sleep spindles. Eur. J. Neurosci. 33, 511-520.

Clemens, Z., Weiss, B., Szucs, A., Eross, L., Rásonyi, G., and Halász, P. (2009). Phase coupling between rhythmic slow activity and gamma characterizes mesiotemporal rapid-eyemovement sleep in humans. Neuroscience 163, 388-396.

De Gennaro, L., and Ferrara, M. (2003). Sleep spindles: an overview. Sleep Med. Rev. 75, 423-440.

Diekelmann, S., and Born, J. (2010). The memory function of sleep. Nat. Rev. Neurosci. 11, 114-126.

Ego-Stengel, V. R., and Wilson, M. A. (2010). Disruption of rippleassociated hippocampal activity during rest impairs spatial learning in the rat. Hippocampus 20, 1-10.

Eichenbaum, H. (2004). Hippocampus: cognitive processes and neural representations that underlie declarative memory. Neuron 44, 109-120.

Fell, J., and Axmacher, N. (2011). The role of phase synchronization in memory processes. Nat. Rev. Neurosci. 12, 105-118.

Fell, J., Fernandez, G., Lutz, M. T., Kockelmann, E., Burr, W., Schaller, C., Elger, C. E., and Helmstaedter, C. (2006). Rhinal-hippocampal connectivity determines memory formation during sleep. Brain 129, 108-114.
Fell, J., Klaver, P., Lehnertz, K., Grunwald, T., Schaller, C., Elger, C. E., and Fernandez, G. (2001). Human memory formation is accompanied by rhinal - hippocampal coupling and decoupling. Nat. Neurosci. 4, 1259-1264.

Fell, J., Staedtgen, M., Burr, W., Kockelmann, E., Helmstaedter, C., Schaller, C., Elger, C. E., and Fernández, G. (2003). Rhinal-hippocampal EEG coherence is reduced during human sleep. Eur. J. Neurosci. 18, 1711-1716.

Ferrara, M., and De Gennaro, L. (2011). Going local: insights from EEG and stereo-EEG studies of the human sleep-wake cycle. Curr. Top. Med. Chem. 11, 2423-2437.

Frankland, P. W., and Bontempi, B. (2005). The organization of recent and remote memories. Nat. Rev. Neurosci. 6, 119-130.

Gais, S., and Born, J. (2004). Declarative memory consolidation: mechanisms acting during human sleep. Learn. Mem. 11, 679-685.

Girardeau, G., Benchenane, K., Wiener, S. I., Buzsáki, G., and Zugaro, M. B. (2009). Selective suppression of hippocampal ripples impairs spatial memory. Nat. Neurosci. 12, 1222-1223.

Girardeau, G., and Zugaro, M. (2011). Hippocampal ripples and memory consolidation. Curr. Opin. Neurobiol. 21, 452-459.

Giuditta, A., Ambrosini, M. V., Montagnese, P., Mandile, P., Cotugno, M., Grassi Zucconi, G., and Vescia, S. (1995). The sequential hypothesis of the function of sleep. Behav. Brain Res. 69, 157-166.

Hobson, J. A., and Pace-Schott, E. F. (2002). The cognitive neuroscience of sleep: neuronal systems, consciousness and learning. Nat. Rev. Neurosci. 3, 679-693.

Huber, R., Ghilardi, M. F., Massimini, M., and Tononi, G. (2004). Local sleep and learning. Nature 430, 78-81.

Jensen, O., and Lisman, J. E. (2005). Hippocampal sequence-encoding driven by a cortical multi-item working memory buffer. Trends Neurosci. 28, 67-72.

Ji, D., and Wilson, M. A. (2007). Coordinated memory replay in the visual cortex and hippocampus during sleep. Nat. Neurosci. 10, 100-107.

Kemp, N., and Bashir, Z. I. (2001). Longterm depression: a cascade of induction and expression mechanisms. Prog. Neurobiol. 65, 339-365.

Langheim, F. J., Murphy, M., Riedner, B. A., and Tononi, G. (2011). Functional connectivity in slow-wave sleep: identification of synchronous cortical activity during wakefulness and sleep using time series analysis of electroencephalographic data. $J$. Sleep Res. 20, 496-505.

Le Van Quyen, M., Staba, R., Bragin, A., Dickson, C., Valderrama, M. Fried, I., and Engel, J. (2010). Largescale microelectrode recordings of high-frequency gamma oscillations in human cortex during sleep. J. Neurosci. 30, 7770-7782.

Lee, A. K., and Wilson, M. A. (2002). Memory of sequential experience in the hippocampus during slow wave sleep. Neuron 36, 1183-1194.

Lewis, P. A., and Durrant, S. J. (2011). Overlapping memory replay during sleep builds cognitive schemata. Trends Cogn. Sci. (Regul. Ed.) 15, 343-351.

Lisman, J. E., and Idiart, M. A. (1995). Storage of 772 short-term memories in oscillatory subcycles. Science 267 1512-1515.

Llinas, R., and Ribary, U. (1993). Coherent $40-\mathrm{Hz}$ oscillation characterizes dream state in humans. Proc. Natl. Acad. Sci. U.S.A. 90, 2078-2081.

Mann, C., Simmons, J., Wilson, C., Engel, J., and Bragin, A. (1997). EEG in human hippocampus, amygdala and entorhinal cortex during REM and NREM sleep. Sleep Res. 26, 27.

Marshall, L., and Born, J. (2007). The contribution of sleep to hippocampus dependent memory consolidation. Trends Cogn. Sci. (Regul. Ed.) 11, 442-450.

Marzano, C., Ferrara, M., Mauro, F., Moroni, F., Tempesta, D., Cipolli, C., and De Gennaro, L. (2011). Recalling and forgetting dreams: theta and alpha oscillations during sleep predict subsequent dream recall. J. Neurosci. 31, 6674-6683.

Massimini, M., Huber, R., Ferrarelli, F., Hill, S., and Tononi, G. (2004). The sleep slow oscillation as a traveling wave. J. Neurosci. 24, 6862-6870.

Massimini, M., Tononi, G., and Huber, R. (2009). Slow waves, synaptic plasticity and information processing: insights from transcranial magnetic stimulation and high-density EEG experiments. Eur. J. Neurosci. 29, 1761-1770.

McClelland, J. L., McNaughton, B. L., and O'Reilly, R. C. (1995). Why there are complementary learning systems in the hippocampus and neocortex: insights from the successes and failures of connectionist models of learning and memory. Psychol. Rev. 102, 419-457.

Mehta, M. R. (2007). Corticohippocampal interaction during up-down states and memory consolidation. Nat. Neurosci. 10, 13-15.

Mölle, M., and Born, J. (2011). Slow oscillations orchestrating fast oscillations and memory consolidation. Prog. Brain Res. 193, 93-110.

Mölle, M., Marshall, L., Gais, S., and Born, J. (2002). Grouping of spindle activity during slow oscillations in human non-rapid eye movement sleep. J. Neurosci. 22, 10941-10947.

Molle, M., Yeshenko, O., Marshall, L., Sara, S. J., and Born, J. (2006). Hippocampal sharp wave-ripples linked to slow oscillations in rat slowwave sleep. J. Neurophysiol. 96, 62-70.

Moroni, F., Nobili, L., Curcio, G., De Carli, F., Fratello, F., Marzano, C., De Gennaro, L., Ferrillo, F., Cossu, M., Francione, S., Lo Russo, G., Bertini, M., and Ferrara, M. (2007). Sleep in the human hippocampus: a stereoEEG study. PLoS ONE 2, e867. doi:10.1371/journal.pone.0000867

Moroni, F., Nobili, L., Curcio, G., De Carli, F., Tempesta, D., Marzano, C., De Gennaro, L., Mai, R., Francione, S., Lo Russo, G., and Ferrara, M. (2008). Procedural learning and sleep hippocampal low frequencies in humans. Neuroimage 42, 911-918.

Moroni, F., Nobili, L., De Carli, F., Massimini, M., Francione, S., Marzano, C., Proserpio, P., Cipolli, C., De Gennaro, L., and Ferrara, M. (2012). Slow EEG rhythms and inter-hemispheric synchronization across sleep and wakefulness in the human hippocampus. Neuroimage 60, 497-504.

Moser, E. I., Krobert, K. A., Moser, M. B., and Morris, R. G. (1998). Impaired spatial learning after saturation of long-term potentiation. Science 281, 2038-2042.

Nadel, L., and Moscovitch, M. (1997). Memory consolidation, retrograde amnesia and the hippocampal complex. Curr. Opin. Neurobiol. 7, 217-227.

Nielsen, T. A. (2000). Mentation in REM and NREM sleep: a review and possible reconciliation of two models. Behav. Brain Sci. 23, 851-866.

Nir, Y., Staba, R., Andrillon, T., Vyazovskiy, V., Cirelli, C., Fried, I., and Tononi, G. (2011). Regional slow waves and spindles in human sleep. Neuron 70, 153-169.

Nobili, L., De Gennaro, L., Proserpio, P., Moroni, F., Sarasso, S., Pigorini, A., De Carli, F., and Ferrara, M. (2012). Local aspects of sleep: observations from intracerebral recordings in humans. Prog. Brain Res. [in press]. 
Nobili, L., Ferrara, M., Moroni, F., De Gennaro, L., Lo Russo, G., Campus, C., Cardinale, F., and De Carli, F. (2011). Dissociated wakelike and sleep-like electro-cortical activity during sleep. Neuroimage 58, 612-619.

O'Keefe, J., and Recce, M. L. (1993). Phase relationship between hippocampal place units and the EEG theta rhythm. Hippocampus 3, 317-330.

Poepel, A., Helmstaedter, C., Kockelmann, E., Axmacher, N., Burr, W., Elger, C. E., and Fell, J. (2007). Correlation between EEG rhythms during sleep: surface versus mediotemporal EEG. Neuroreport 18, 837-840.

Siapas, A. G., and Wilson, M. A. (1998). Coordinated interactions between hippocampal ripples and cortical spindles during slow wave sleep. Neuron 21, 1123-1128.

Spoormaker, V. I., Czisch, M., Maquet, P., and Jäncke, L. (2011). Large-scale functional brain networks in human non-rapid eye movement sleep: insights from combined electroencephalographic/functional magnetic resonance imaging studies. Philos. Transact. A Math. Phys. Eng. Sci.369, 3708-3729.
Spoormaker, V. I., Schröter, M. S., Gleiser, P. M., Andrade, K. C., Dresler, M., Wehrle, R., Sämann, P. G., and Czisch, M. (2010). Development of a large-scale functional brain network during human nonrapid eye movement sleep. J. Neurosci. 30, 11379-11387.

Steriade, M. (2003). The corticothalamic system in sleep. Front. Biosci. 8, d878-d899.

Steriade, M., and Timofeev, I. (2003). Neuronal plasticity in thalamocortical networks during sleep and waking oscillations. Neuron 37, 563-576.

Steriade, M., Timofeev, I., and Grenier, F. (2001). Natural waking and sleep states: a view from inside neocortical neurons. J. Neurophysiol. 85, 1969-1985.

Tononi, G., and Cirelli, C. (2006). Sleep function and synaptic homeostasis. Sleep Med. Rev. 10, 49-62.

Tse, D., Takeuchi, T., Kakeyama, M., Kajii, Y., Okuno, H., Tohyama, C., Bito, H., and Morris, R. G. (2011). Schema-dependent gene activation and memory encoding in neocortex. Science 333, 891-859.
Van Der Werf, Y. D., Altena, E., Schoonheim, M. M., Sanz-Arigita, E. J., Vis, J. C., De Rijke, W., and Van Someren, E. J. (2009). Sleep benefits subsequent hippocampal functioning. Nat. Neurosci. 12, 122-123.

Wagner, T., Axmacher, N., Lehnertz, K., Elger, C. E., and Fell, J. (2010). Sleep-dependent directional coupling between human neocortex and hippocampus. Cortex 46, 256-263.

Wamsley, E. J., Tucker, M., Payne, J. D. Benavides, J. A., and Stickgold, R. (2010). Dreaming of a learning task is associated with enhanced sleepdependent memory consolidation. Curr. Biol. 20, 850-855.

Wang, G., Grone, B., Colas, D., Appelbaum, L., and Mourrain, P. (2011). Synaptic plasticity in sleep: learning, homeostasis and disease. Trends Neurosci. 34, 452-463.

Watts, D. J., and Strogatz, S. H. (1998). Collective dynamics of "small-world" networks. Nature 393, 440-442.

Wieser, H. G. (1984). “Temporal lobe epilepsy, sleep and arousal: stereoEEG findings," in Epilepsy, Sleep and Sleep Deprivation, eds R. Degen, and E. Niedermeyer (Amsterdam: Elsevier Science Publishers B.V.), 137-167.

Conflict of Interest Statement: The authors declare that the research was conducted in the absence of any commercial or financial relationships that could be construed as a potential conflict of interest.

Received: 16 November 2011; accepted: 28 March 2012; published online: 17 April 2012.

Citation: Ferrara M, Moroni F, De Gennaro L and Nobili L (2012) Hippocampal sleep features: relations to human memory function. Front. Neur. 3:57. doi: 10.3389/fneur.2012.00057

This article was submitted to Frontiers in Sleep and Chronobiology, a specialty of Frontiers in Neurology.

Copyright (c) 2012 Ferrara, Moroni, De Gennaro and Nobili. This is an openaccess article distributed under the terms of the Creative Commons Attribution Non Commercial License, which permits non-commercial use, distribution and reproduction in other forums, provided the original authors and source are credited. 